\title{
Sensor Validation and Fieldbus
}

\section{by Manus Henry}

Intelligent sensors are now able to generate diagnostic messages, and Fieldbus can carry them, but how are they to be integrated into a control system? It is argued that a standard format must be adopted, and on-line uncertainty is proposed as a metric for data quality.

$\mathbf{M}$

anagers of industrial plant find themselves under increasing pressure from opposing directions. One the one hand there is the ever-expanding legislation concerning safety, and in particular environmental protection, and on the other hand there is the relentless drive for improved productivity and reduced costs. Sensor validation is an area of technological development offering enhanced performance from both perspectives.

Here the term "sensor" is used to describe a complete measurement device including transducer and transmitter, while "validation" is taken to mean the assessment of sensor performance. Sensor validation can lead to fewer accidents and spillages, thereby enhancing safety, and provide better product quality and reduced unsheduled shutdowns, improving plant efficiency and availability.

\section{Sensor Fault Detection}

Work on sensor fault detection began in the late 1950's when it became clear that automated control based on faulty data could lead to disaster. The classical approach has been to apply mathematical modelling, or more recently knowledge-based techniques, to spot inconsistencies in data from several sensors. Typically plant-specific software is written to run alongside the control system.

Although fault detection theory has attracted much academic interest, it has rarely been applied in the process industries. The reasons are both technical and economic. Technically it has proved difficult to develop plant models that are reliable and robust to plant disturbances. Economically, such schemes are expensive, as each is unique, and must be created and maintained by highly-skilled personnel.

Increasingly, intelligent sensors are being designed with a self-diagnosing capability. Diagnostic and measurement data can both be communicated via a digital communication link such as Fieldbus.

While there are technical advantages to this approach (each sensor can monitor internal signals for evidence of faults, or use active self-testing), the main advantages are economic. Each sensor manufacturer can spread the cost of developing diagnostics over many customers. On the other side, with many users now concentrating on their core business, it is attractive to buy a fault detection capability off-the-shelf, rather than support in-house expertise. 
But what form should diagnostic data take, and how is it to be generated and interpreted? This article describes the SEVA (SEnsor VAlidation) scheme, developed by Oxford University, Foxboro GB Ltd and ICI, in a collaboration which began in 1989 .

\section{Fault Descriptions}

A sensor may be viewed in different ways: to the service engineer it is a plant component that must be maintained, while to the operator it is a source of measurement data.

Currently, most intelligent sensors which provide diagnostics generate a device-specific fault code or message (e.g. 'fault 43 - fouled membrane'). For maintenance purposes a simple error code is probably an adequate description of the fault.

Determining the appropriate operational response to a sensor fault is more complex (see Fig. 1). Detailed knowledge is required both from the sensor manufacturer (to determine what the fault is and how it affects the measurement) and from the operator or control engineer (to determine the impact on plant operation of the loss of measurement quality, and to select an appropriate operational response).

Note that 'measurement quality' rather than the sensor diagnosis is needed to determine the impact on the control system. This is because plant operation is not dependent upon the sensor itself, but rather the measurement that it generates. There is in general a complex relationship between a sensor fault and the resulting measurement error (if any). This relationship will become increasingly complex as sensors and their diagnostics become more sophisticated. For example, if a sensor generates several different measurements, then a single fault will in general affect each measurement differently. This relationship between faults and measurements will of course vary between sensor technologies and will be at least slightly different for comparable products from different vendors.

If a vendor only provides a device-specific error code then the user is left the job of mapping from sensor fault to measurement consequence. However few users (if any) have access to the required vendor's knowledge of sensor faults and measurement quality. By far the most suitable delivery vehicle for this knowledge is the sensor itself, which can carry out a detailed analysis of the current fault condition to derive the impact on each of its measurements.

\section{The Validity Index}

An alternative approach to error codes is for the sensor to generate a 'Validity Index' (VI) for each measurement, which describes the validity or quality of that measurement. The VI can be used by the control system to take operational decisions in

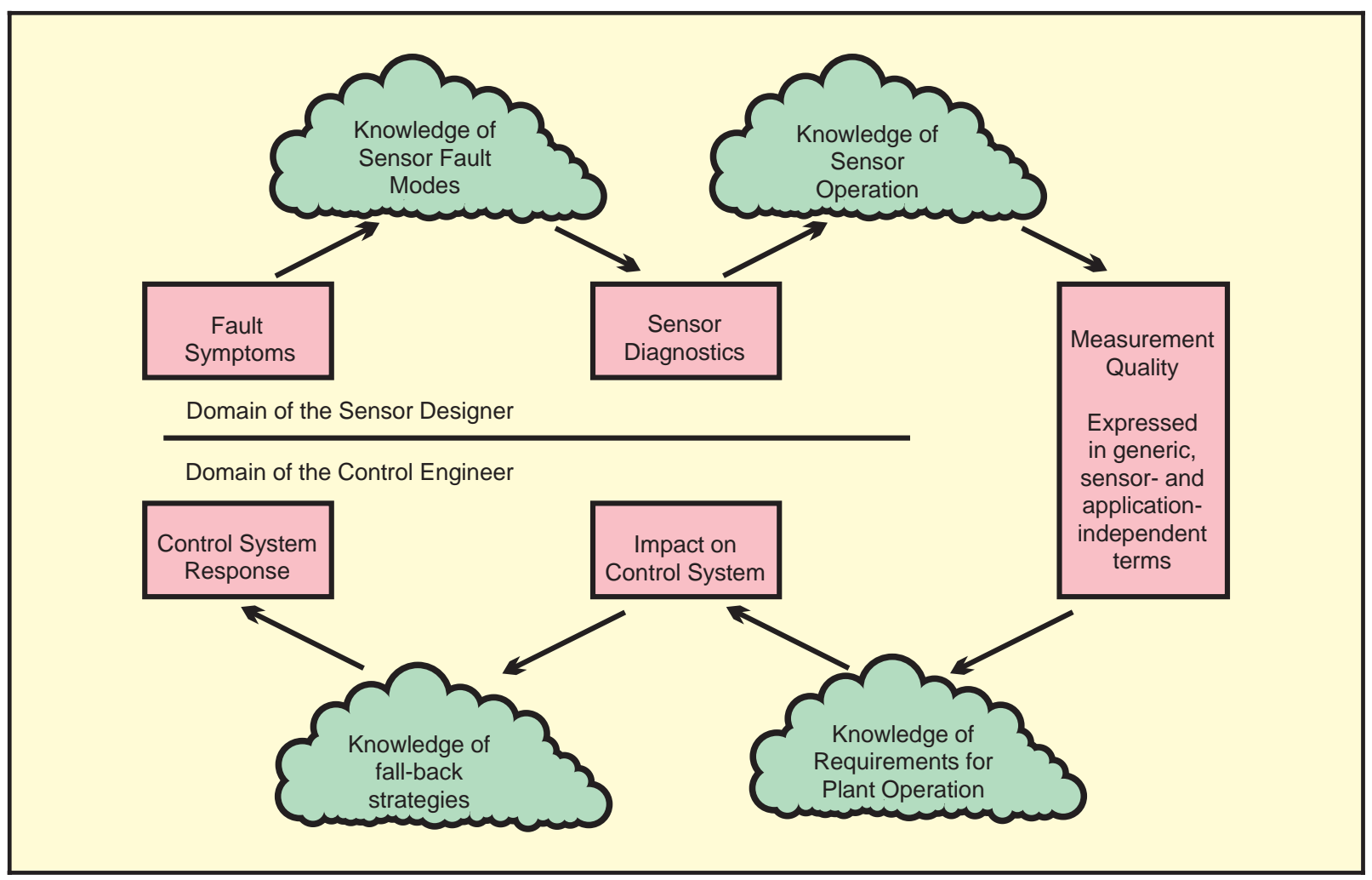

Fig. 1 Knowledge sets needed to determine control system response to sensor fault symptoms 
response to any sensor fault.

To avoid the difficulties associated with error codes, it is desirable that the VI should be generic, that is entirely independent of the sensor type or vendor. The control system could then be configured to respond to changes in measurement quality irrespective of the particular sensor technology. A subsequent exchange of, say, a magnetic flow meter for a vortex meter, should require no modification to the fault accommodation strategy.

Whatever form the VI might take, it should be easily understood so that its generation and interpretation are straightforward. It should be sufficiently expressive to be able to distinguish between different degrees or levels of validity. Even more importantly, it must be consistent and in some sense verifiable.

Another desirable feature of the VI is that we should be able to calculate the validity of a derived measurement (Fig. 2). In this way we can have validity data for every plant parameter of interest, even those calculated indirectly from sensor data.

If the VI is truly to reflect measurement quality, then there are many factors other than the absence or presence of sensor faults that also have a bearing. The most obvious example is the sensor technology itself. We might reasonably expect the flow data from the latest vortex meter to be 'better' than that from a dp cell and orifice plate. Other factors might include the time since the last calibration, or process noise, or even installation effects. Such a VI would convey useful information all the time and not just when a sensor fault occurs.

A final issue to consider is whether the sensor is to adopt a corrective strategy. Suppose it detects a fault should it convey faulty measurement data or should it provide a best estimate of the true process value by compensating for the fault? In each case the role of the VI is different. In the former case the VI simply indicates that the data is faulty, while in the latter it must indicate that the data has been corrected. In either case the VI may also give some more general indication of measurement 'quality'.

\section{The Single Bit VI}

The simplest form of VI is a single bit to indicate whether the measurement is 'valid' or 'invalid'. This has the appeal of simplicity and universality - it can

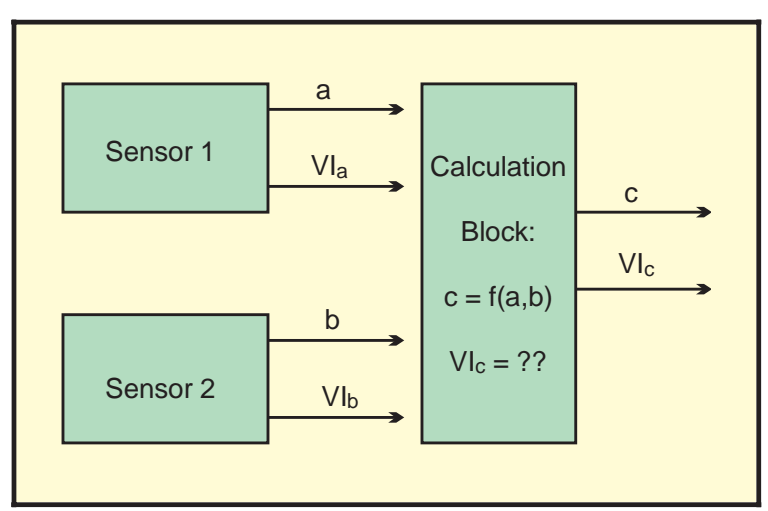

Fig. 2 Propagation of Validity Indices certainly be applied to any measurement.

Of course many of the desirable features of the VI could not be accommodated in a single bit scheme. There could be no general method for combining validities, nor any accounting for factors such as measurement technology. It would not be possible for the sensor to use a corrective strategy. These drawbacks might seem light compared with the advantage of having a simple scheme.

Further consideration reveals a more serious problem. If the validity bit is to be employed consistently, it must be interpreted by the control system as 'use/do not use this data'. In assigning a value to the validity bit, the sensor itself is deciding whether or not the measurement is 'usable'. Clearly, while this is a function of the sensor fault, it also depends upon the application. The single-bit approach is thus the opposite extreme of using error codes (see Fig. 1) - the sensor designer is being asked to decide without the necessary application knowledge.

For example, if a pressure cell develops a minor fault (e.g. loss of the temperature compensation), its measurement data may still be adequate for, say, monitoring water supply pressure, but it would probably be inadequate for feedback control in a hazardous application. The sensor cannot be expected to know what its data is to be used for, and so it cannot judge the utility of the data for the application.

\section{The SEVA Scheme}

We will now describe the form of VI proposed by the SEVA Research group at Oxford ${ }^{1}$. The starting point is the adoption of a corrective strategy. The sensor is required to provide a best estimate of the process measurand at all times, even in the presence of a sensor fault, along with a VI to indicate the estimated quality of that estimate.

A SEVA sensor employs self-diagnostics, but provides extra stages of processing. If a fault occurs its impact on each measurement is assessed, and the measurement is corrected if necessary. Validity indices are generated which describe the resulting quality of each measurement in generic, device-independent terms. These enable the control system to make an appropriate response to the sensor fault irrespective of the particular sensor technology or supplier. This is of course not possible using today's device-specific error codes. 
Figure 3 shows the parameters generated by the SEVA instrument. There are two validity indices generated for each validated measurement value or VMV (corresponding to the conventional measurement). These are called the Validated Uncertainty (VU) and the Measurement Value Status or MV Status. Additionally, a single Device Status is generated, which summarises the physical health of the sensor itself. Of course, a detailed, device-specific diagnosis of any fault is always available for the maintenance engineer.

\section{Validated Uncertainty}

Uncertainty is a well-established engineering concept. For any measurement $x$, the associated uncertainty, $\delta x$, expresses a reasonable bound for the measurement error, at a given level of probability. In other words, we might expect the true value of the measurand to lie within the uncertainty region surrounding the measurement, that is within $x \pm \delta x$.

Uncertainty is defined and applied in numerous national and international standard documents. In particular, it is used to quantify error when tracing the calibration of an individual instrument back to known reference standards. In custody transfer applications, for example, it is frequently a requirement that an instrument of known uncertainty (assuming strict limits on process behaviour and ambient conditions) is used to meter the process fluid.

An uncertainty value takes into account a number of factors affecting the accuracy of the measurement, including measurement technology, calibration error, installation effects and environmental effects. Traditionally uncertainty has been a static analysis, assigning a single uncertainty value to an instrument. In the SEVA scheme, the uncertainty of each measurement is calculated every sample, to allow for dynamic effects. Note that as the values are calculated

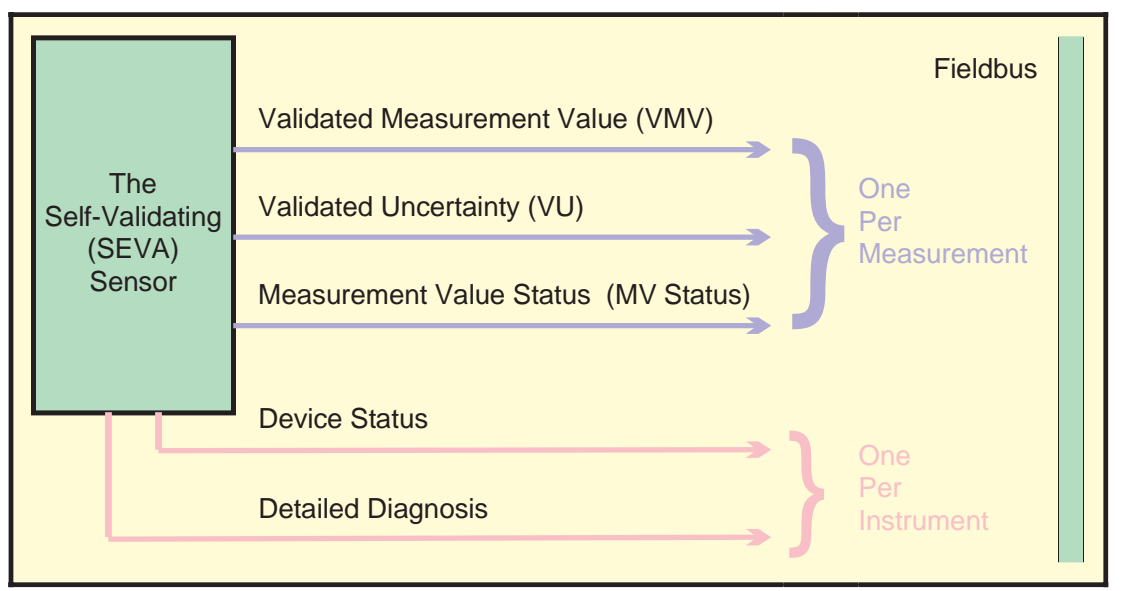

Fig. 3 Sensor Validity Parameters in real time with changing process values, separation of uncertainty into bias and precision terms, as described in certain standards, is not appropriate. The definition of uncertainty is further extended to include the impact of any sensor faults on the measurement.

The standards provide a rule for calculating the uncertainty of arbitrary functions. Given the uncertainties of $\mathrm{x}$ and $\mathrm{y}$, say, we can calculate the uncertainty of $\mathrm{R}$ using the following sum of squares formula:

$$
\delta_{R}^{2}=\left[\frac{\partial R}{\partial x}\right]^{2} \delta_{x}^{2}+\left[\frac{\partial R}{\partial y}\right]^{2} \delta_{y}^{2}
$$

The Validated Uncertainty provides the major indicator of the quality of the Validated Measurement Value. The VMV is a 'best estimate' of the current value of the measurand. Under normal conditions it is calculated using the latest data from the transducer, but this is not always the case. If a minor fault affects the sensor then a correction may be applied, and if a major fault occurs then the VMV may be calculated by projection from historical data. While the VU will increase to accommodate the reduced accuracy of such estimates, by itself it cannot indicate that the current VMV is, say, based on historical rather than live data. This information may be important to the control system, which would not wish to continue feedback control based only on historical data. A second, discrete, validity index is also generated with each VMV to inform the control system in effect "how this VMV was calculated', and this parameter is called the Measurement Value Status.

\section{Measurement Value Status}

There are six possible values of the MV Status, corresponding to six different scenarios for how the VMV has been generated. The principal values are:

- CLEAR indicates that there is no fault, and that the VMV has been calculated normally from the latest transducer data.

- BLURRED indicates that the measurement has been partially impaired by the presence of a sensor fault, and that a correction has been applied in the calculation of the VMV. The VU is increased appropriately to indicate the reduced accuracy of the estimate. 
- BLIND indicates that a diagnosed fault has occurred which has a severe impact upon the measurement, and so the current VMV is projected from historical data, not live transducer data. A BLIND measurement should never be used for feedback control.

- DAZZLED is a temporary status used when transducer data is clearly erroneous, but there is insufficient internal evidence to confirm that a substantial fault has occurred. The current VMV is projected from historical data, but the expectation is that the internal diagnosis will soon be resolved and that the status will then switch to one of the other values. DAZZLED is used to deal with the occurrence of temporary but severe effects such as a spikes. It would, for example, be undesirable for a control loop to be switched to manual in response to the controlled measurement turning BLIND and then, only a few seconds later, the measurement were to return to CLEAR.

The two additional states are as follows:

- SECURE indicates that the VMV has been generated from redundant transducers or sensors, all of which are in nominal condition. This status is useful in critical applications where the user needs the reassurance that even if one transducer or sensor fails, CLEAR data will still be available.

- UNVALIDATED indicates that validation has not been in operation in the sensor which generated the measurement.

Note that it is not only sensor 'faults' which may have a detrimental impact upon a measurement. A self-test, for example, may for a short time have a partial or severe effect on the measurement. In such circumstances the strategies for calculating the VMV, VU and MV status are identical, for it is the quality of the measurements which matter to the control system, rather than the underlying cause of any degradation in quality.

\section{Prototypes}

A number of SEVA prototypes are being developed at Oxford, based upon industrial sensors manufacturered by Foxboro. These include temperature, dissolved oxygen, and pressure sensors. A particularly interesting subject for validation is the coriolis mass flow meter, as it generates three independent measurements: temperature, density and mass flow respectively. The PC-based SEVA prototype can detect a number of fault modes. In each case, it corrects each measurement and provides updated uncertainty and status parameters in real time. An example of its output is shown in Fig. 4.

The temperature, density and mass flow measurements (VMVs) are shown with their respective uncertainty intervals shaded in light blue. The status of each measurement is indicated both by the colour of the VMV and the text in the corresponding panel on the left. In this particular example, a serious fault has occurred in the temperature probe. The fault has been detected and the temperature status is set to BLIND. Temperature data is still provided, projected from past behaviour, but the reducing confidence in this estimate is indicated by the increasing uncertainty. As the temperature measurement is used in the calculation of both density and mass flow, these two measurements are set to BLURRED. The increasing uncertainty in temperature is propagated into the uncertainy equations for density and mass flow, resulting in appropriate increases. This example is described in more detail in reference, which demonstrates that without SEVA functions enabled, the fault results not only in an unusable temperature measurement, but also erroneous offsets of $20 \%$ and $5 \%$ in density and mass flow respectively.

Fig. 4 Output from SEVA Coriolis Mass Flow Meter 


\section{SEVA and Fieldbus}

The SEVA scheme assumes the availability of intelligent sensors and a digital communications capability. The SEVA metrics are an attempt to improve intelligent sensor interoperability by describing measurement validity in generic terms. These ideas are thus ideally suited to the standardisation arena, and to Fieldbus in particular, which so far has given fairly limited consideration to validation issues. The SEVA team have been active in promoting dialogue on validation in a number of forums. Of course these issues are independent of any particular protocol: we are members of the WorldFIP and P-Net user associations, as well as the Fieldbus Foundation User Advisory Council. In addition, we have been involved in the European project EIAMUG (described elsewhere in this issue), and made numerous presentations to interested groups, such as the BP Fieldbus trials consortium.

We are also concerned with developing our own Fieldbus demonstration capability, and to this end we have been creating a new laboratory at Oxford, illustrated in Fig. 5. The modular flow rig can support a number of sensors, each of which is validated in an industrial PC, held in the rack on the right of the picture. Each PC is provided with a Fieldbus capability, so that SEVA metrics can be communicated to a control PC. In addition, SEVA metrics are sent to a Foxboro IA series control system. The rig will be used for developing control strategies and higher level fault detection techniques, assuming the availability of SEVA metrics.
P-Net, the Danish National Standard, has been used for the first fieldbus network, due in part to price and availability, but primarily because of the associated software packages Hugo and Vigo. These provide easy access to Fieldbus variables (e.g. data in a remote instrument) for any Windows application supporting OLE2 automation. Such applications include most spread-sheets and Visual Basic.

The simplest demonstration of SEVA in a Fieldbus context is simply to communicate SEVA metrics from one Fieldbus node to another, and this has been our first application. The hardware and software architectures are shown in Figure 6. One industrial PC hosts a thermocouple validation card ${ }^{2}$. This card has been created using hardware compilation techniques $^{3}$ to perform all aspects of thermocouple validation within integrated circuits, and demonstrates that sensor validation could be implemented in ASICs. It generates SEVA metrics which may be accessed via the bus of the host PC.

Within the Windows environment, two software packages access this data. The first is National Instrument's Labview software, which has been configured to provide an on-line graphical display of the validated thermocouple data. The second is a simple Visual Basic program which collects the SEVA data and sends it via VIGO, HUGO and the P-Net to the other PC. In the second PC the process is reversed. The Visual Basic program is the same in each PC, operating either in transmitter mode (top $\mathrm{PC}$ ) or in monitor mode (lower PC). Figure 7 shows the two PCs in operation.

Fig. 5 SEVA and Fieldbus Laboratory at Oxford 


\section{Conclusion}

This paper has discussed the need for generic descriptions of measurement quality generated by intelligent sensors, outlined the concept of the Self-Validating Sensor, and demonstrated the transmission of SEVA data over a commericial Fieldbus.

\section{References}

1 HENRY, M. P. and CLARKE, D. W.: The Self-Validating Sensor: Rationale, Definitions and Examples. Control Engineering Practice, 1993, 1, (4), pp 585 - 610.

2 ATIA, M., BOWLES, J., CLARKE, D.W., HENRY, M.P., PAGE, I., RANDALL, G. and YANG, J.C.: A Self-Validating Temperature Sensor Implemented in FPGAs. FPGA'95 Conference, Oxford, Auguest 1995.

3 HENRY, M.P.: Keynote Paper: Hardware Compilation - A New Technique for Rapid Prototyping of Digital Systems - Applied to Sensor Validation.Control Engineering Practice, 1995, 3, (7), pp 907 -924.

(C) IEE: 1995

The author is with the Engineering Science Department, Oxford University, Oxford OX1 3PJ. This work has been sponsored by Foxboro GB Ltd and the EPSRC. Thanks to Proces Data for the loan of P-Net equipment.

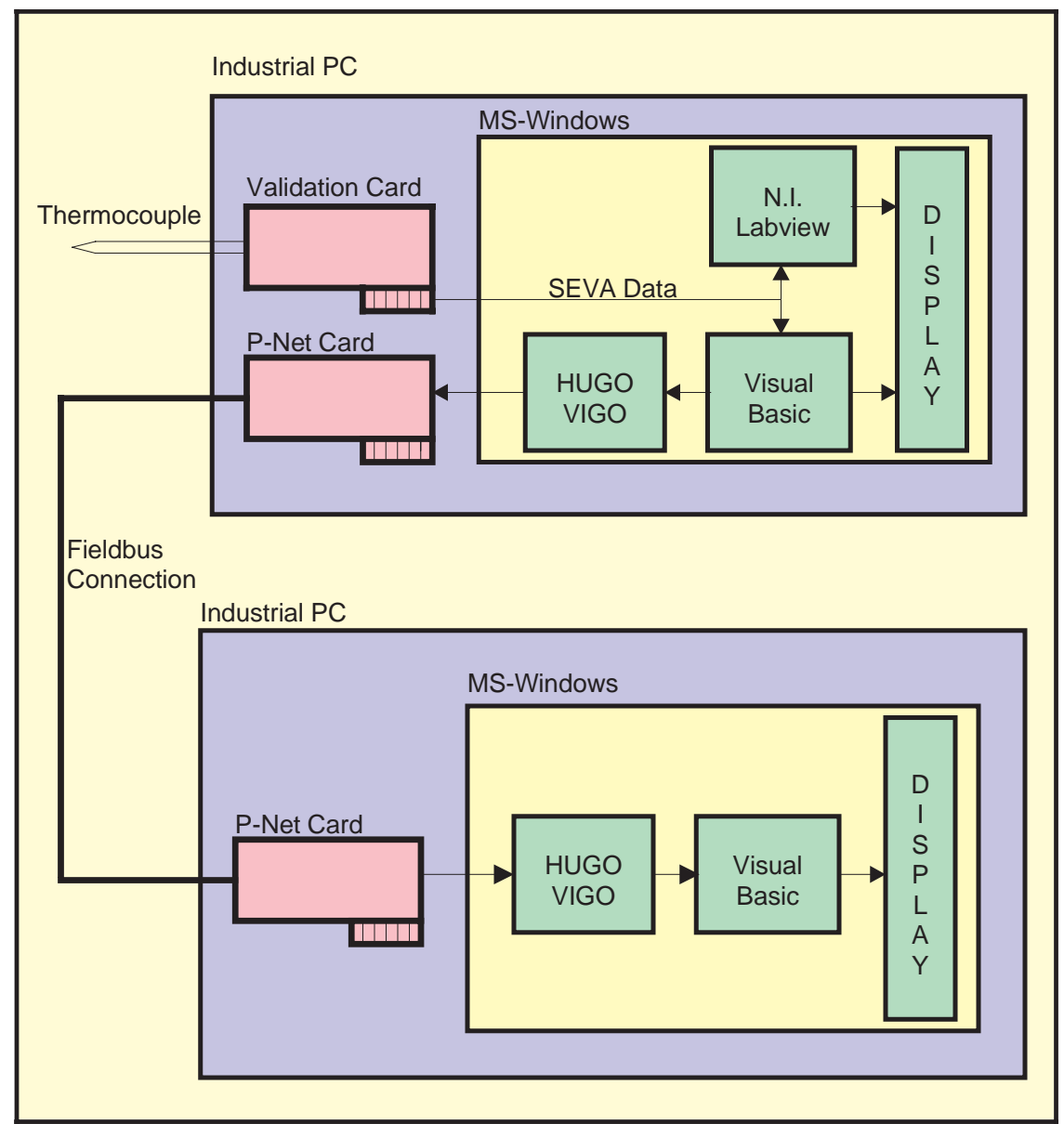

Fig. 6 Architecture for transmission of SEVA data over fieldbus

Fig. 7 Industrial PCs communicating SEVA metrics via P-Net. 